\title{
Geschichtliches über den Schmerz in Pathologie, Nervenphysiologie und Mentalität
}

\author{
Von Antoinette Stettler
}

\section{Einleitung}

Dem Schmerz, der zur Erfahrung jeden Lebens gehört, wurde selbstverständlich schon in der Antike Aufmerksamkeit geschenkt. Er fand schließlich seinen Platz im wohlabgerundeten galenischen System des Pneuma psychikon als Reizung des Tastsinnes, die im Herzen ihren Widerhall hat. Das Phänomen Schmerz wurde, eines unter vielen anderen, als Sinneserfahrung aufgefaßt und war an einem relativ unauffälligen Ort in das großartige Gebäude des psycho-physiologischen Menschenbildes eingebettet. Um 1550 beginnt sich diese Anschauungsweise zu verändern. 1554 publizierte Jean Fernel unter dem Titel «Physiologia» seine Lehre von der Funktion des Menschen, die zwar immer noch Psychisches und Somatisches mitumfaßte, aber in der Titulatur eine Neuheit darstellte: Physiologie - die Lehre von der Funktion des Körpers. 1590 trat Rudolf Göckel mit einem Werk «Psychologia (hoc est de perfectione hominis)» an die Öffentlichkeit. Auch dieser Titel war ein Novum, auch wenn das Augenmerk, wie ersichtlich, nicht auf Psychologie im heutigen Sinne, sondern auf die sittliche Verbesserung des Menschen zielte. Diese beiden Titel sollen andeuten, daß mit der Namengebung die Gebiete des Körpers und der Seele eigenständige Untersuchungsgebiete zu werden begannen. Der körperliche und der seelische Bereich des Menschen wurden gesondert thematisiert.

An der Thematisierung zweier bisher in der Generallehre des Pneuma psychikon verwobenen Erkenntnisbereiche läßt sich die Brüchigkeit der überlieferten Systematik ablesen. Die Thematisierung - um im Bild zu sprechen - wirkt sich wie ein Mikroskop aus, welches einzelne Untersuchungsgegenstände vergrößert, d.h. auch eigenständig erscheinen läßt. Physiologie und Psychologie beginnen, autonom zu werden.

In diesen Zusammenhang gehört auch, daß das Phänomen Schmerz ebenfalls in der zweiten Hälfte des 16. Jahrhunderts zum eigenen Thema wurde. Zur Bestätigung dieses Sachverhaltes sollen Dissertationen dienen, die in Straßburg und in Basel unter dem Vorsitz von Theodor Zwinger I verteidigt wurden. Es gelte auch, daß Felix Platter in seiner «Praxis» (Basel 
1601-1611) eine der drei Hauptabteilungen mit «De doloribus» überschrieben hat. Auch das Thema Schmerz wurde somit vergrößert.

Die Thematisierung einzelner Wissensgebiete im 16. Jahrhundert hat verschiedenerlei Gründe. Mit der Erfindung der Druckerpresse erstens erhöhte sich die Möglichkeit, nicht nur die ganz wichtigen Werke großer Gelehrter zu vervielfältigen und zu verbreiten, sondern auch Werke junger Autoren wie Dissertanden konnten in einem größeren Kreise zu Kenntnis gebracht werden. Auch in anderen Gebieten ist für die genannte Zeit die Thematisierung bestätigt.

Zweitens mag speziell die Problematik des Schmerzes nicht ausschließlich Domäne der Medizin gewesen sein. Diese mag sie, wahrscheinlich zu ungleichen Hälften, mit der Kirche geteilt haben.

«Horum accidentium consideratio cum et Physici sit et Theologici et Ethici; nos tamen de ea tamquam Medici ad fines artis ... pertinere potest.»

(Während die Betrachtung dieser Zustände [der Schmerzen] die Naturkundigen, Gottesgelehrten und Sittenlehrer angeht, kann sie die Ärzte dennoch zur Verbesserung ihrer Kunst betreffen.) ${ }^{1}$

Mit dem ebenfalls seit 1550 festzustellenden Aufbrechen der religiösen Formen in Renaissance und Reformation hat die Vorstellung des Schmerzes als Heimsuchung Gottes an Leuchtkraft einzubüßen begonnen. Die Erforschung der natürlichen Gegebenheiten des Schmerzes rückte zunehmend in den Vordergrund.

Auch eine rein medizinische Ursache für das Interesse am Schmerz ist drittens ins Feld zu führen. Ebenfalls in derselben Zeit stellen wir fest, daß bei den medizinischen Lehrbüchern die poetischen und gravitätischen Titel, wie sie das arabistische Mittelalter liebte, zu Gunsten der handgreiflicheren wie «Praxis» und dgl. verschwinden. Darin kündet sich eine mehr pragmatische Ausrichtung der Medizin an. Auch das Interesse am Schmerz entstammt der pragmatischen Dimension. Es ist deshalb beinahe ein Truismus zu sagen, daß ein so ausgesprochen pragmatisch orientierter Arzt wie der erwähnte Platter gerade eben in seinem Lehrbuch «Praxis» eine Abteilung mit «De doloribus» betitelt.

Das Phänomen Schmerz stellte nun die Ärzte vor eine doppelte Aufgabe. Einerseits mußte es in ein weniger brüchiges System der Psycho-Physiologie eingegliedert, andererseits aber auch in einer praktisch orientierten Krankheitslehre - in einer «Pathologia», ebenfalls seit Jean Fernel 1555 ein eigenständiges Wissensgebiet - plaziert werden. 


\section{Der Stellenwert des Schmerzes in der Krankheitslehre}

Seit Andreas Vesals «Fabrica» (1543) und seit Jean Fernels «Pathologia» (1555), beides Werke, die sich von der Theorie der vorangegangenen Jahrhunderte zu entfernen trachteten und die physische Sichtbarkeit der menschlichen Dinge in den Vordergrund stellten, wurde der Ort der Krankheit zunehmend häufiger im krankhaft veränderten anatomischen Substrat gesucht und auch gefunden.

War es seit altersher klar, daß die sogenannten Krankheiten «solutionis continuitatis», d.h. die Wunden, die Knochenbrüche und Verrenkungen, Schmerzen verursachen konnten, wurde es seit Vesal und Fernel immer augenfälliger, daß der Schmerz in den Körperhöhlen in vielen Fällen einem Geschwür, einer Geschwulst oder dergleichen entsprochen haben mußte. Der stechende, bohrende, grelle usw. Schmerz findet, konjugiert mit dem durch Autopsie gefundenen galenischen «locus affectus», einen Platz im Krankheitsverstehen:

«Prioris efficiens causa sunt ea omnia quaecumque pungunt, dissecant, divellunt, contundunt aut erodunt sive externa ea sint aut interna.»

«... alles, was sticht, schneidet, teilt, stößt oder nagt, sei es außen oder innen.»²

Die dergestalt umschriebene Ursache des Schmerzes dient Platter dazu, in seiner vorgenannten «Praxis» erstmals eine systematische Zuordnung der Schmerzzustände zu ihrem jeweiligen pathologisch-anatomischen Substrat vorzunehmen: Schmerzen mit deutlicher autoptischer Lokalisation gehören im Brustbereich der Pleuritis und Pneumonie an, im Bauchbereich dem Ulcus und der Peritonitis, am Bewegungsapparat der Gicht und dem Rheumatismus. Selbstverständlich kommen dazu die schmerzenden Hautkrankheiten, Verletzungen und Brüche, so wie sie schon immer zu den Krankheiten «solutionis continuitatis» gezählt wurden und sichtbar waren (im Schema durchgehend fein unterstrichen). Zu den Schmerzen ohne äußerlich oder bei der Autopsie sichtbaren Veränderung gehören die vagen Schmerzzustände bei allen Fiebern (im Schema unterbrochen unterstrichen) sowie diejenigen der Hypochondrien (im Schema fett unterstrichen).

Seine pragmatische Einteilung der Schmerzen sieht im einzelnen folgendermaßen aus: 


\section{Schmerzen}

1. die überstarken Erregungen der Sinnesorgane (Auge, Ohr, Geschmack, Geruch)

2. die unbestimmt lokalisierten Schmerzen bei Fiebern

3. Kopfweh

4. Augenschmerz (Konjunktivitis usw.)

5. Ohrenweh (Otitis media usw.)

6. Schmerzen der Nase (Furunkel usw.)

7. Schmerzen der Mundregion

8. Zahnweh

9. Schmerzen im Rachen (Angina usw.)

10. Schmerzen im Thorax ( Pneumonie, Pleuritis)

11. Herzschmerzen

12. Schmerzen in den Hypochondrien

13. Bauchschmerzen ( Peritonitis, Ulcus)

14. und 15. Schmerzen im Genital- und Afterbereich

16. Schmerzen im Bewegungsapparat (Rheuma, Arthritis)

17. Schmerzen an der Körperoberfläche ${ }^{3}$

Ein großer Teil der Schmerzen konnte auf diese Art im organischen Substrat dingfest gemacht werden. Mit andern Worten: der Schmerz wurde objektiviert. Es versteht sich von selbst, daß für den Prozeß der Verknüpfung Schmerz = Läsion mit der Entwicklung der pathologischen Anatomie sich auch immer neue Möglichkeiten ergaben. Seit 1650 ungefähr sind denn auch zahlreiche Monographien entstanden, die im Titel den Ausdruck «-algia» führen, Cardialgia, Coxalgia usw.

Es muß hier betont werden, daß Platters Einteilung der gesamten Krankheiten, die neben den Funktionsstörungen und den Verunstaltungen die Schmerzen als eine der drei Erscheinungsweisen der Krankheit überhaupt, eine absolute Neuheit darstellte. Obwohl die Krankheitssystematiker des 17. Jahrhunderts wie Daniel Sennert, Johannes Jonston und François de le Boe Platter und sein neues System ausdrücklich erwähnen, übernehmen sie die Emphase, mit welcher er den Schmerz belegte, nicht. Man muß die Nachwirkungen der Voreingenommenheit gegenüber der pragmatischen Bedeutung des Schmerzes vielmehr in zahlreichen Dissertationen, die namentlich auch in Platters Stadt Basel entstanden, sehen. In die Krankheitslehren drang der Schmerz erst im 18. Jahrhundert. 
Indessen geschah im 17. Jahrhundert etwas anderes. Die Konjunktion des Schmerzes mit dem Ort der Krankheit, die in so vielen Fällen mit an Sicherheit grenzender Wahrscheinlichkeit gegeben war, machte den Blick frei für eine andere Art von Schmerz - nennen wir ihn den für den Arzt und Pathologen elusiven Schmerz. Er ist wohl auch identisch mit Platters Schmerz in den Hypochondrien. Anders ausgedrückt, blieben die subjektiven Schmerzzustände übrig, die sich mit keinem oder die sich noch nicht mit einem «locus affectus» in Verbindung bringen ließen. Der elusive Schmerz kann zwar sehr stark sein, aber er «lügt». ${ }^{4}$

Dieser elusive Schmerz muß derart ins Bewußtsein mindestens der gebildeten Stände gedrungen sein, daß Molière in seinem «Malade imaginaire» (1673) das leidende Individuum, dessen «Einbildung» die übelsten Qualen hervorruft, der allgemeinen Lächerlichkeit preisgeben konnte. Das Schauspiel wurde populär und ist es bis heute geblieben.

Auch in vor allem englischen Ärztekreisen zeigt sich ein großes Interesse an solchen Schmerzzuständen. Wir erinnern an Thomas Sydenhams Monographie über die Hysterie/Hypochondrie (1682). Er macht darin auf ebensolche Schmerzerscheinungen aufmerksam, die er offensichtlich häufig beobachtet hatte, die sich aber in irgendeiner Weise nicht regelrecht verhielten, d.h. nicht mit einer Läsion in Zusammenhang bringen ließen.

Sed nec spectabilior est huius morbi frequentia, quam varietas illa multiformis qua se ostendit, et nullos fere aemulatur ex iis affectibus quibus atteruntur miseri mortales ${ }^{5} . »$ «Nichts indessen fällt mehr auf als die Häufigkeit dieser Krankheit, auffällig auch die vielfältige Unterschiedlichkeit, unter welcher sie auftritt und die doch beinahe keine jener Krankheiten nachahmt, von welchen die erbärmlichen Sterblichen zerschlissen werden»

Zur Beschreibung gerade schwerster hysterischer Schmerzzustände gebraucht Sydenham das Wort «mentiri». ${ }^{4}$ Während Molière in solchen Fällen insinuiert, es sei der Mensch, der lüge und damit den Schmerz in die Sittlichkeitslehre verweist, wo er bis heute unter anderem auch geblieben ist, ordnet ihn Sydenham in die Krankheitslehre als Teilaspekt seiner Krankheit Hysterie/Hypochondrie ein.

Haben diese Kranken gerade keinen Schmerzanfall, sind sie von allerlei Mißbehagen geplagt (dejectio spirituum) und leiden unter herabgesetzter Allgemeinbefindlichkeit ${ }^{6}$. Der elusive Schmerz weist demnach das ganze Spektrum vom physisch betonten bis zum mehr psychisch schattierten Zustand auf. Es liege ihm eine Ataxie des Pneuma psychikon folgerichtig zu Grund ? 
Wir nannten soeben als Teilbefund der Hysterie die herabgesetzte Allgemeinbefindlichkeit. Es ist dabei zu betonen, daß damit keineswegs die Verschlechterung des Allgemeinbefindens und die Schmerzzustände bei den Fiebern gemeint waren. Vielmehr machte gerade im 17. Jahrhundert die Differenzierung der Fieberkrankheiten wesentliche Fortschritte.

Steht am Beginn des 17. Jahrhunderts Platters revolutionäre Erhebung der Schmerzen als Krankheitskategorie, so sind wir am Anfang des 18. mit der Differenzierung der Schmerzen in drei Aspekte konfrontiert:

1. lokalisierbaren oberflächlichen oder durch Autopsie sichtbar gemachten läsionsbedingten Schmerz

2. den Schmerz bei fieberhaften Krankheiten der verschiedensten Genese

3. den Schmerz, der nach Sydenham «lügt» und der dem Krankheitsbild der Hysterie/Hypochondrie zugeordnet wird.

Platters «Praxis» war beileibe nicht die einzige Krankheitslehre des 17. Jahrhunderts, aber sie blieb nachweislich bis gegen 1720 recht populär. Nach 1731 machte sich nun François Boissier de Sauvages an die Überarbeitung der Krankheitslehre, um in der Weise des 18. Jahrhunderts die reichen Früchte der vorangegangenen Zeit unter ein einziges Dach zu bringen. An ihn und Carl Linné (1763) schließen die meisten der systematischen Krankheitslehren des 18. Jahrhunderts an.

Es gilt hier nicht, Boissier de Sauvages' System als Ganzes mit demjenigen von Platter zu vergleichen. Wir greifen einzig den Stellenwert heraus, den bei ihm die Schmerzen haben, und stellen fest, daß sie eine unter zehn Krankheitskategorien bilden. Boissier de Sauvages kennt zwar die schmerzverursachenden Fieber; sie sind aber unter dem Aspekt der Temperaturerhöhung ein eigenes Kriterium. Befand Platter also die Fieber als eine Kategorie des Schmerzes, so sah Boissier de Sauvages den Schmerz in sinnvollerer Weise als einen Teil der fiebrigen Erkrankung, so wie wir es heute noch sehen ${ }^{8}$.

Im übrigen kannte Boissier de Sauvages «dolores vagi», die aber nicht dem Sydenhamschen lügenden Schmerz entsprechen, sondern eher eine Ahnung des funktionellen Schmerzes im modernen Sinn darstellen.

Mit Linnés «Genera morborum» (1763) finden wir uns an einer weiteren Bruchstelle der Schmerzauffassung. Zwei Jahre zuvor, 1761, trat Giovanni Baptista Morgagni mit «De sedibus morborum ...» an die Öffentlichkeit, einem Werk, das die Übersicht über alle bis dahin bekannten lokalisierbaren Krankheitszustände gibt. Damit war nun aber auch auf einen Schlag der 
lokalisierbare Schmerz kein prinzipielles pathologisches Problem mehr. Die Bedeutung der Arbeit Morgagnis für die Pathologie ist bekannt genug, so daß hier eben nur gewissermaßen ihr Nebeneffekt erwähnt zu werden braucht: die endgültige Unterordnung des Schmerzes unter die Läsion.

Im Linnéschen System bildet nun der Schmerz eine Unterabteilung der Nervenkrankheiten: Linné meint offensichtlich nur noch den allgemeinen und auch den nicht läsionsbedingten Schmerz und hält ihn für eine Störung des Urteilsvermögens. Damit wird gleichzeitig der psychische Anteil des Schmerzgeschehens ins Licht gerückt. Durch Linnés Betrachtungen wird klar, daß der Schmerz recht eigentlich nur etwas über den Zustand des Nervensystems aussagt.

William Cullen seinerseits, 1768, wollte vom Schmerz als relevantem Diagnosekriterium überhaupt nichts mehr wissen. Unter seinem Begriff der Neurose verstand er nicht nur die allgemeinen und vagen, sondern auch die imaginären, also hysterisch-hypochondrischen Schmerzzustände; ja er wollte geradezu alle Krankheitszustände als Neurose, als nervös bedingt aufgefaßt wissen ${ }^{9}$.

In ungefähr dieselbe Zeit fällt auch die Prägung des Begriffes Neuralgiedes Nervenschmerzes sui generis - durch den Arzt François Chaussier (1746-1828). Nicht nur Nervensystem und Urteilsvermögen waren die Ursache aller Schmerzen, sondern die Nerven selbst waren es, die schmerzen konnten ${ }^{10}$.

Während Linné den Schmerz ganz allgemein als Störung des Urteilsvermögens und damit des Nervensystems, Cullen ausschließlicher als Ding der gestörten Nervenfunktion sah, blieben andere Autoren des 18. Jahrhunderts konservativer. Rudolf August Vogel ${ }^{11}$ und Johann Baptist Michael Sagar ${ }^{12}$ führen den Schmerz noch als eines unter elf bzw. zwölf Diagnosekriterien an. Trotzdem darf behauptet werden, daß seit rund 1770 ein großer Teil der Schmerzen einen Krankheitshinweis im Sinne des «locus affectus» geworden waren; ein anderer Teil einen Krankheitshinweis, welcher der Temperaturerhöhung bei den Fiebern zugeordnet war, darstellte; und schließlich ein Restbestand von Schmerzen übrig blieb, der mit Cullen als elusiv der nervösen Störung der Krankheit Hysterie/Hypochondrie als zugehörig betrachtet wurde.

Um 1800 stehen wir demnach vor der Situation, daß sich der Schmerz von der generellen Krankheitskategorie, wie wir sie bei Platter gefunden haben, in ein untergeordnetes Krankheitszeichen einerseits und in eine noch nicht näher beschriebene Nervenempfindung andererseits gewandelt hatte. Der 
Schmerz hatte in der praktischen Krankheitslehre demnach seine Doppelstellung gefunden, die er heute noch einnimmt. Es folgt die Aufgabe, die geschichtliche Entwicklung der Erforschung der Nervenempfindung nachzuzeichnen.

\section{Der Stellenwert des Schmerzes in der Psychophysiologie}

Man ist heute ohne weiteres gewöhnt, Sinnesempfindung und somit auch Schmerz mit Kenntnisnahme und Erfahrung über die Außenwelt gleichzusetzen. Schmerzerleben vermittelt nun aber meist nur indirekte Erfahrung über die Außenwelt. Bei einer Verletzung z.B. erleben wir im Schmerz zunächst nur unsere eigene körperliche Befindlichkeit. Erst durch einen von den anderen Sinnen vermittelten Rationalisierungsprozeß erfahren wir, was in der Außenwelt geschah. Ähnlich der propriozeptiven «Sinnesempfindung» möchten wir den Schmerz als ein Erleben verstanden wissen, das auf der Grenze zwischen der Außenwelt und der Innerlichkeit der eigenen Erfahrung stattfindet. Wenn wir nun einige Betrachtungen zur Geschichte der Erforschung der Nervenempfindung anstellen, so müssen wir einerseits den Darstellungen der Innenwelt, anderseits auch denjenigen der Grenze zur Außenwelt nachgehen. Immer waren solche Entwürfe auch mit dem Versuch verknüpft, einen Ort für die betreffende Empfindung bzw. das betreffende Erleben zu finden, einen Ort, an welchem die Funktion geschieht.

Es fällt auf, daß noch Platter im Zusammenhang mit der Überreizung der Sinne nur deren vier: Gesicht, Gehör, Geruch und Geschmack, nennt. Der Tastsinn fehlt. Nun ist aber die menschliche Person von der Außenwelt so eindeutig durch die Haut und deren Sinne abgegrenzt, daß es erstaunen muß, wenn diese elementare Tatsache keiner näheren Bewußtwerdung unterzogen wurde. Während sich die klassische Antike schon mit Ausführlichkeit der Erforschung der sogenannt höheren Sinne, dem Auge und dem Ohr, zuwandte, galt ihr die Haut einerseits als Mantel des Körpers, andererseits als globales Sinnesorgan, das zwar «ohne Fenster» gewisse Erkenntnis der Außenwelt vermittelte. Diese spiegelte sich, wenn sie mehr mit dem Gefühl verbunden war, im Herzen. Der Verstand enthielt, wie die platonische Philosophie sagte, die angeborenen Ideen und war nur lose im «sensus communis» mit der Außenwelt verbunden. Der Ort des «sensus communis» fand sich irgendwo im Kopf ${ }^{13}$. 
Diese summarische Zusammenfassung über die Vorstellung des Verhältnisses von Außenwelt und Innerlichkeit in der Zeitspanne, als der Schmerz als Thema aktuell wurde, muß ergänzt werden. Als Ausgangspunkt nehmen wir das überlieferte galenisch/arabische System der Psychophysiologie, wie sie zuhanden der Ärzte bei Theodor Zwinger I um 1580 in Basel gelehrt wurde.

«Der menschliche Körper und alle seine empfindlichen Teile sind mehr oder weniger dem Schmerz unterworfen. Es handelt sich entweder um eine Belästigung des Tastsinnes, des traurigen Gefühls oder der traurigen Empfindung.»

(Materia doloris subjecta est corpus humanum secundum omnes partes suas sensiles, magis, minus. Forma est ipsae sensus tactus molesta et tristis affectio, sive tristis sensatio). ${ }^{14}$

Diese Aussage ist zwar sinngemä $\beta$, aber unzulänglich übertragen, weil sie in einem uns nicht mehr geläufigen Sinnzusammenhang steht. Sie ist eingebettet in die aristotelische Lehre von den vier Urgründen, der causa materialis, der causa formalis, der causa efficiens und der causa finalis. In unser heutiges Verstehen übersetzt, wäre die «materia doloris», d.h. die causa materialis des Schmerzes sein Ort im Körper; die «forma doloris», d.h. die causa formalis seine Äußerung als Überreizung des Tastsinnes. Die causa efficiens, die Ursache, so wie wir es heute verstehen, haben wir zuvor erwähnt: «... alles, was sticht, schneidet, zerreißt ... usw.» Wir fügen als causa finalis des Schmerzes die Absicht bei, mit welcher der Kranke Hilfe z. B. beim Arzt sucht. Was der Text nicht erwähnt, dem damaligen Leser aber selbstverständlich, ist, daß die Empfindung und das Gefühl zur Sphäre des Pneuma psychikon gehörten. Dieses wäre demnach die Wirkkraft auch des Schmerzes, eine Kraft, die gleichmäßig und gleichzeitig den Tastsinn und die Empfindung belebt. Zur Veranschaulichung dieser Schmerztheorie diene das folgende Diagramm:

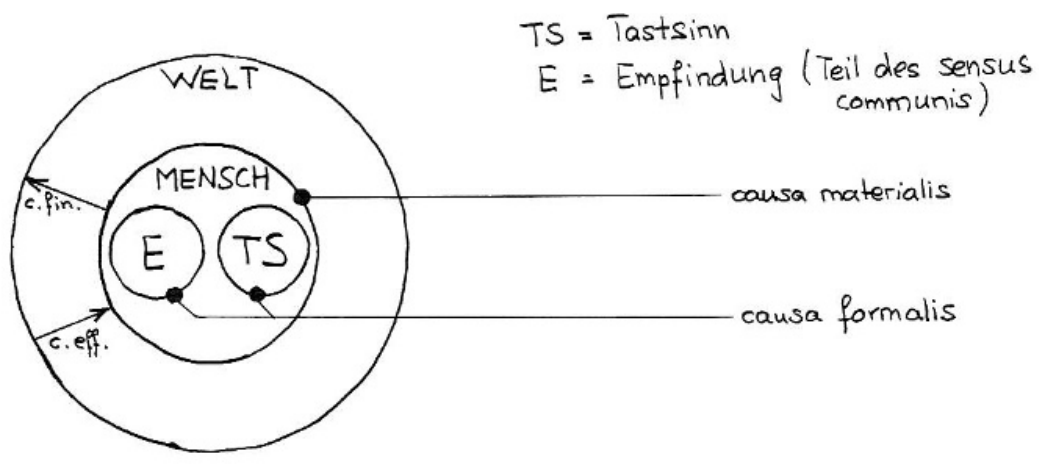


Die vier causae des Schmerzes sind einander gleichgeordnet. Durch diese Gleichgewichtigkeit ergibt sich eine abgerundete, in sich selbst ruhende Anschauung. Tastsinn und traurige Empfindung, beides Teile der causa formalis, sind nicht getrennt. Wir konstatieren keinen ausgesprochenen Unterschied zwischen Außengrenze der Innenwelt und Innerlichkeit, wie wir ihn heute mit der Vorstellung von Sinnesempfindung und Wahrnehmung haben.

Die Gültigkeit der aristotelischen Kausallehre kam um 1550 ins Schwanken, als im Gefolge der Experimente Galileis nurmehr die causa efficiens als Ur-sache zu gelten begann. Durch das «Cogito ergo sum» des Descartes

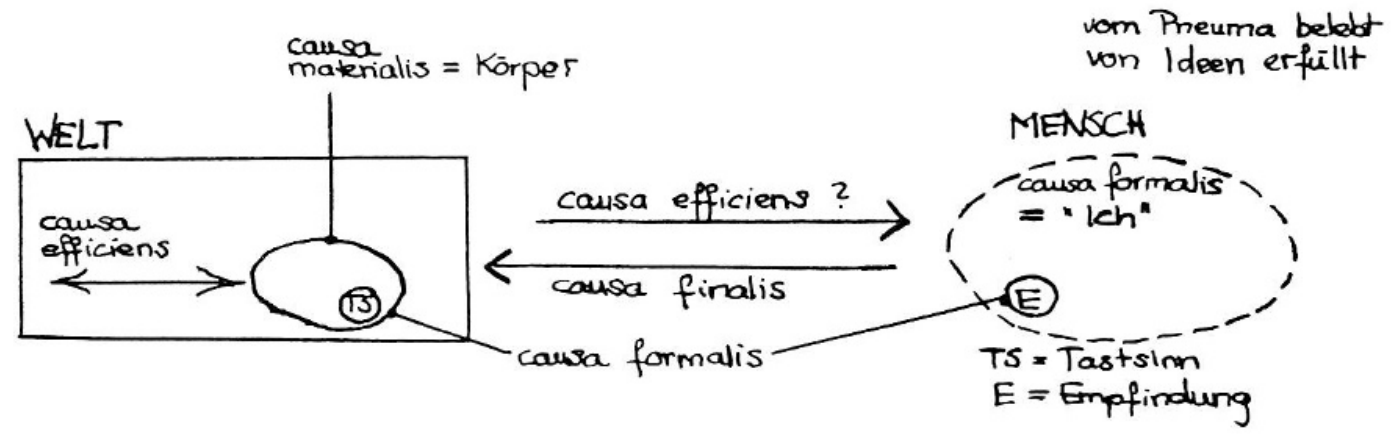

(1637) bekam außerdem die Denkwirklichkeit, die Ich-Wahrnehmung, der Existenzbeweis mittels Erlebens der eigenen Person ein geradezu mystisches Ausmaß ${ }^{15}$. Hat Descartes alles Körperliche einerseits in die Domäne des mechanisch funktionierenden Uhrwerks, d.h. in die Domäne der causa efficiens verwiesen, so gab er andererseits dem Individuum durch das denkerische Ich-Erleben ein derartiges Übergewicht. Weil es für uns heute außerordentlich schwierig geworden ist, uns die Veränderung vorzustellen, die das cartesianische Denken für den aristotelisch orientierten Menschen bedeutet haben muß, vergleichen wir folgendes Diagramm mit dem vorangegangenen:

Weil der Körper des Menschen nunmehr gänzlich zur Welt gehörte und er in der gleichen Weise wie die ballistischen Maschinen des Galilei, den causae efficientes unterlag, konnte nicht ausbleiben, daß er auch nach solchen Regeln untersucht wurde, was ausgiebig in der Iatrophysik geschah. Die causa materialis ihrerseits erhielt durch anatomische, schließlich mikroskopische Untersuchungen ebenfalls eine erhöhte Autonomie. Was aber für unsere weitere Argumentation am wichtigsten erscheint, ist die Tatsache, daß sich die causa formalis, zuvor zu gleichen Teilen Tastsinn und Wahrneh- 
mung, nun zu spalten begann in eine gar bis zum Existenzbeweis erhobene Ich-Komponente und zum im Körper, d.h. in der Welt, liegenden Tastsinn. Der Tastsinn erhält Brückenfunktion zwischen Ich-Erleben und Welt. Der Mensch hat sich einesteils erweitert, indem er seinen Körper in die Außenwelt entließ, sich anderenteils aber auch verengt, indem er sich auf sein Ich verwiesen sah.

Die Folge dieser Umschichtung zeigt sich in John Lockes «Essay on Human Understanding ...» (1796). Locke, der große, vor allem auch experimentelle Erfahrung in der Naturkunde und Medizin seiner Zeit besaß, faßte das sich seit 1600 abzeichnende neue psychophysiologische Gedankensystem gegen sein Lebensende dergestalt zusammen, daß eine neue Erkenntnistheorie überhaupt entstand. In seinem «Essay» räumt er radikal mit den platonischen, dem Menschen eingeborenen Ideen auf. Das Innen ist bei der Geburt, wie er es nennt, ein unbeschriebenes Blatt Papier. Die Sinnesempfindungen, «sensations» bekommen nun eine ganz überragende Wichtigkeit. Hatten sie bei Descartes mehr eine Brückenfunktion zwischen der Außenwelt und dem Ich-Bewußtsein, so wurden sie für Locke die einzige Quelle, die im Laufe des Lebens das gänzlich leere Innen durch die im Hirn stattfindende Wahrnehmung (perception) mit Ideen füllte.

"This great source of most of the ideas we have, depending wholly upon our senses, and derived by them to the understanding, I call sensation". ${ }^{16}$

Wenden wir uns noch einmal den Diagrammen zu, würden die Verhältnisse bei Locke so aussehen:

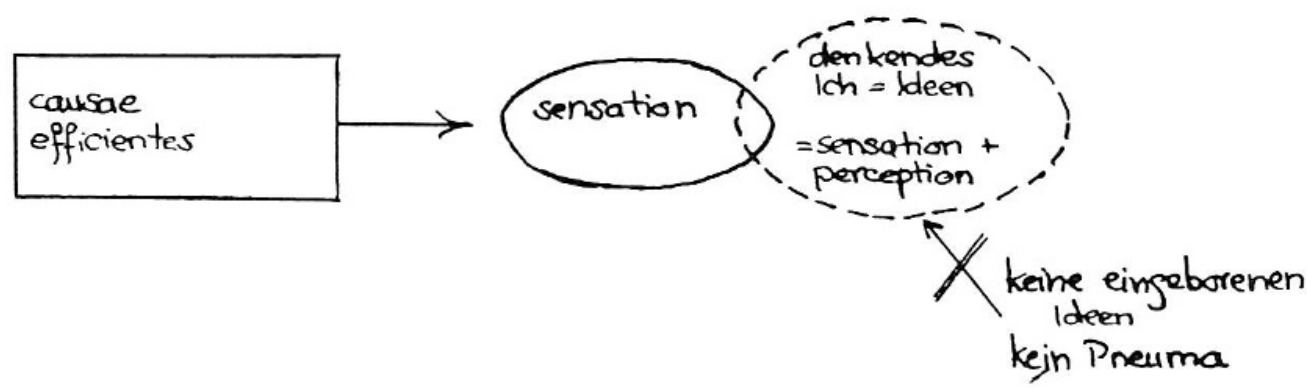

Von der Auffassung des 16. Jahrhunderts bleibt nicht mehr viel übrig. Von den vier Urgründen gilt nur noch einer mit Namen, die causa efficiens in der Außenwelt. Wollte man die Sache strapazieren, könnte man sagen, daß sich die vier causae in eine Kette gebildet hätten, dergestalt, daß die causa efficiens auf die causa materialis (Körperwelt), diese auf die causa formalis 
(Sinn und Verstand) einwirke und diese wiederum die causa finalis (Wille) hervorrufe. Nur hätte diese Kette ein an Wichtigkeit alles andere überragende Glied, nämlich den zur Sensation gewordenen Teil der causa formalis. Eine solche Rückübersetzung dient allerdings nur dazu, der Neuartigkeit von Lockes Denken erst recht Relief zu geben. Ohne Übertreibung kann gesagt werden, daß mit Locke die Bewußtwerdung der Sinnesempfindung und der Wahrnehmung stattfand, und es bleibt festzustellen, daß wir auch heute keine grundsätzlich andere Sicht der Dinge haben.

\section{Abriß der Physiologie des Nervensystems}

1. Das körperliche Substrat der Sinnesempfindung, wir wissen es heute, liegt im peripheren, dasjenige der Sinneswahrnehmung im zentralen Nervensystem. Wir machen heute, mindestens zu Zwecken der Übersicht und der Darstellbarkeit, genau wie Locke eine Zäsur zwischen der Außengrenze der Innenwelt und der Innenwelt.

Wir haben angedeutet, daß die Auffindung des Ortes der Empfindungsund Gedankenwelt eine uralte Frage des menschlichen Selbsterkennungsprozesses ist. Als durch die anatomischen Forschungen der Medizinschule von Alexandria und zusammengefaßt durch Galen das Gehirn, das Rückenmark und die Nerven immer deutlicher in einem Funktionszusammenhang begriffen wurden, bekam diese Lokalisationssuche die erste Verankerung in der Tatsächlichkeit. Wohl blieb das Herz noch für mehr als ein Jahrtausend der erlebte Ort der Gefühle, immer mehr wurde aber das Gehirn für den Sitz des Verstandes, des «Sensus communis» gehalten. Damit gewannen die mit dem Hirn verbundenen Strukturen, vor allem auch die in der Haut vermuteten Nervenendigungen als Zuleiter zum Gehirn neue Bedeutung. Für die Belange des Schmerzes war die Zuleitung der Tastempfindung von Wichtigkeit. Avicenna schon nannte den Zeigefinger den «Iudex in mensurationem».

Die Kenntnislage der Funktion des Nervensystems und damit der Schmerzempfindung stellt sich um 1550 etwa wie folgt dar: die Haut, der mit Poren und Nervenendigungen versehene Mantel des Körpers, vermittelte über die ebenfalls porös gedachten Nervenstränge und das Rückenmark mit Hilfe des Pneuma psychikon dem Gehirn die Tastempfindungen, zu denen auch der Schmerz gehörte. Im Gehirn trafen solche Empfindungen das «sensorium commune», welches durch das nun peripherwärts fließende 
Pneuma die verschiedensten Tätigkeiten hervorrief, z. B. Wegziehen betroffenen Körperteils von der Schmerzquelle oder Reiben der schmerzenden Stelle, usw.

Das erneute Interesse der Renaissance an der Sinneserfahrung ganz allgemein und am Bau des Nervensystems im besondern führte zu wichtigen Erkenntnissen im Bereich des Gesichtssinnes (Platter zur Netzhautfunktion; Kepler zur Sehoptik) und der Muskelbewegung (Fernel zur unwillkürlichen Bewegung), blieb aber für die Erforschung des Tastsinnes zunächst ergebnislos.

Neues geschah für die Erforschung des Tastsinnes erst, als Marcello Malpighi, der mit dem Mikroskop arbeitete, in seiner «Epistula de externo sensus tactus organo» (1665) glaubte, im Stratum germinativum der Haut die Tastorgane erkannt zu haben. Es ist für den weiteren Verlauf der Geschichte nicht so wichtig, daß Malpighis Körperchen sich als am Tastsinn nicht beteiligt erwiesen; das Entscheidende bleibt, daß Malpighi ein Organ des Tastsinnes propagierte und damit die Suche einleitete, die über Abraham Vater (1741), Filippo Pacini (1842), Georg Meissner, Friedrich Merkel und Angelo Ruffini (1920 ff.) dann zur Differenzierung der verschiedenen in der Haut sich befindenden Sinnesorgane führte. Zu erwähnen bleibt auch Ernst Heinrich Weber, dessen Arbeiten seit 1830 soviel für die Erfassung der Physiologie der Hautsinne beigetragen haben ${ }^{17}$, für den aber der Schmerz immer noch eine Funktion der Hautsinnesorgane blieb. Die endgültige Lokalisation der Schmerzempfindung in die freien Nervenendigungen und damit die Erkenntnis, daß der Schmerz eine autonome Empfindung darstellt, die mit dem Tastsinn erst in zweiter Linie zu tun hat, fällt dank der Zusammenarbeit vieler Forscher ins 20. Jahrhundert.

2. Die Suche nach der Schmerzempfindung in der Peripherie, an der Außengrenze der Innenwelt, hat damit ihren vorläufigen Abschluß gefunden. Selbstverständlich fand parallel zu solcher Untersuchung und zum Teil unabhängig davon auch diejenige des Erlebens bzw. der Schmerzwahrnehmung im «sensorium commune», dem Zentrum des Nervensystems, statt. Nicht daß Thomas Willis der einzige Beschreiber des Nervensystems seit Vesal gewesen wäre, aber er ist wohl unbestritten der Bedeutendste. Auf sein Werk «Cerebri anatome» (1664) muß zurückgegangen werden, wenn ein Wendepunkt in der Schmerzauffassung gesucht wird.

Willis, der nicht nur, auf Descartes zurückgreifend, als erster den nervösen Reflex beschrieb, vermutete, daß die Basalganglien des Gehirns, 
seiner Meinung nach vor allem das Corpus striatum», eine wichtige Funktion in der Verarbeitung der Sinnesempfindung und damit des Schmerzes haben. Ebenfalls meinte er, daß von diesen Strukturen wiederum Impulse in die Peripherie gehen, die dort dann Muskelreaktionen hervorrufen. Schmerz ist für ihn vorwiegend mit dem Tastsinn verbunden, obwohl er an anderer Stelle auch von der schmerzempfindlichen Nervenfaser spricht. Interessant sind seine Überlegungen zur zentralen Schmerzverarbeitung. Dabei unterscheidet er drei Stufen der Bewußtwerdung des Schmerzes. Ein schmerzhafter «Stimulus» mag erstens nur bis ins Corpus striatum gelangen und dort die besagte Muskelreaktion auslösen, er mag zweitens eine schon klarere, aber flüchtige bewußte Vorstellung im Corpus callosum hervorrufen oder drittens in die Region der Beurteilung und ins Gedächtnis in der Großhirnrinde aufsteigen. Die Leitung des «Stimulus» dachte sich Willis lichtschnell, die Reaktion im Muskel als eine Explosion iatrochemischer Natur. Damit ist angedeutet, daß sich Willis die Wirkkraft des ganzen im Nervensystem zentral- und peripherwärts verlaufenden Schmerzgeschehens nicht mehr als das unkörperliche Pneuma psychikon dachte, sondern dieser Kraft schon irgendwelche materiellen Attribute zusprach ${ }^{18}$.

Es bleibt uns anzumerken, daß Locke als Student ein Schüler von Willis war. Daher erstaunt nicht, daß Lockes Philosophie in einem gewissen Sinne die Summa der «Cerebri anatome» darstellt: das Gehirn und damit Verstand, Gedächtnis usw. mußten geradezu das «unbeschriebene Blatt Papier» sein, das über die sinnliche Erfahrung im Laufe des Lebens gefüllt wurde. Gegen das Ende des 17. Jahrhunderts finden wir uns demnach mit Willis und Locke am Berührungsort der Lehre von der Sinnesempfindung ganz allgemein mit der Erkenntnislehre überhaupt. Die Nervenphysiologie ist somit wiederum in den Rahmen der Philosophie gestellt, den Rahmen, den sie bis heute, selbstverständlich unter weiterer Ausfüllung, im wesentlichen beibehalten hat.

3. Seit Willis und dem Oxforder Gelehrtenkreis experimentierten die Physiologen bekanntlich mit der Methode der Subtraktion am anatomischen Substrat, d.h. mit Unterbindungen von Gefäßen, Nerven und mit Exstirpationen. Seit 1730 kam der dekapitierte Frosch als Experimentier«gegenstand» in Gebrauch, dessen Organe und Gewebe mit den verschiedensten Reizen - Schmerzen? - mechanischer, chemischer und seit der zweiten Hälfte des 18. Jahrhunderts elektrischer Art behandelt wurden. Albrecht von Haller indessen machte seine Experimente nicht nur am kopflosen Frosch, sondern am Tier, das sein gesamtes Nervensystem behalten hatte. In 
seinem Werk «De partibus corporis humani sensilibus (oder sensibilibus) et irritabilibus» (1752) kommen wir an die Nahtstelle der Nervenempfindung mit dem Schmerz. Indem er bei seinen Tierversuchen über die empfindlichen und erregbaren Gewebe den Reiz durch Schmerz als Prüfstein, ja geradezu als Definitionsmodul für die sensiblen Teile verwendete, verknüpfte er definitiv den Schmerz mit dem Nervensystem. Das Konzept, daß alles Schmerzende mit der Nervenempfindung zu tun habe, ist seither für die Forschung verankert. Allerdings muß betont werden, daß Haller nicht etwa Schmerzforschung betrieb, um dieser Geißel der Menschheit abzuhelfen, er brauchte vielmehr den Schmerz als Stimulus für das Nervensystem in der experimentellen Situation. Haller befand ferner, daß nicht das Pneuma psychikon, wie es noch Willis beinahe glaubte, schließlich die Sensibilität hervorrufe, sondern eine dem Nervensystem innewohnende Kraft, die L. A. Galvani seit 1780 als die elektrische postulierte und nachwies.

In den von Haller aufgezeigten Bahnen ging die Erforschung der Funktion des Nervensystems im 19. Jahrhundert weiter, indem der Schmerz als Reiz gebraucht und die Reaktionen darauf studiert wurden. Auf diese Weise gelang Charles Bell und François Magendie zwischen 1809 und 1822 der Nachweis der sensiblen Natur der Hinterwurzeln des Rückenmarks. Zusammen mit Johannes Müllers Postulat der spezifischen Sinnesempfindung stellte dieser Nachweis den entscheidenden Impuls zur Erforschung der Funktion des Rückenmarkes dar; ferner mit verbesserten Mikroskopen und histologischer Färbetechnik, welche durch Sichtbarmachung der Neuronen (Nervenzellen) die Lehre vom Reflexbogen untermauerten, verschaffte sich um die Wende zum 20. Jahrhundert die Anschauung Geltung, daß der Tractus spino-thalamicus des Rückenmarks diejenige Nervenbahn sei, welche die Schmerzreize zu einem wenn auch untergeordneten Zentrum des Gehirns leite. Diese Entdeckung ist nun der Umschlagspunkt, wo nicht nur Erforschung der Funktion des Nervensystems aufgrund des Schmerzreizes stattfand, sondern recht eigentlich die Schmerzforschung mit dem Ziel der Schmerzbekämpfung eine theoretische Begründung zu besitzen begann. 1911 fand die erste chirurgische Durchtrennung der antero-lateralen Nervenbahnen des Rückenmarks, d.h. auch des Tractus spino-thalamicus, die sog. «Chordotomie», zu therapeutischen Zwecken statt ${ }^{19}$.

Mit dem Konzept der «gate-control» besitzt die Nervenphysiologie heute eine recht umfassende Erklärung des Schmerzphänomens. Man ist der begründeten Meinung, da $\beta$ es in der Körperperipherie, in den inneren Organen sowie entlang der Blutgefäße bestimmte Schmerzrezeptoren gibt, 
deren anatomisches Substrat zum Nervensystem gehört. Werden diese Nozizeptoren auf bestimmte Art gereizt, leiten die dazu gehörenden Nervenbahnen den dem Rezeptor eigenen Impuls schließlich zu den nervösen Schaltstellen im Thalamus, wo sie entweder gedämpft, unterdrückt oder zur Großhirnrinde weitergeleitet werden und dann als Schmerz bewußt werden (Wall/Melzack 1965 bzw. 1978). Diese gate-control-Theorie, die den komplexen Verschaltungen der Nervenimpulsleitung große Beachtung gibt, erklärt neben den autonom und daher unbewußt ablaufenden Schmerzreaktionen vielfältigster Art auch zum Teil, weshalb das Schmerzerleben individuell so beträchtliche Unterschiede aufweist. Nach Melzack wird zwischen der sensorisch-diskriminativen und der motivational-affektiven Schmerzkomponente unterschieden, was sich recht gut mit der schon immer aufgesuchten Grenze zwischen der Außenwelt und der Ich-Erfahrung deckt. Ähnlich wie um die Wende zum 20. Jahrhundert der Tractus spinothalamicus erweist sich heute der Thalamus, zum phylogenetisch älteren Gehirnteil gehörend, als Drehpunkt der Schmerzverarbeitung, und wie sich gezeigt hat, auch der Schmerzbekämpfung (Enkephalinrezeptortheorie $1978 \mathrm{ff}$.).

4. Es sollen noch einige Bemerkungen zur Schmerzbekämpfung angeführt werden. Die vorhin erwähnte Chordotomie war die erste solcher Maßnahmen, die eine theoretische Begründung in der Nervenphysiologie besaß. Sie war aber beileibe nicht die erste Schmerzbekämpfung überhaupt. Zu allen Zeiten und überall wurde der Schmerz auch mit pharmakologischen Mitteln angegangen. Die Rolle, welche der Alkohol darin gespielt hatte und noch spielt, ist wohl am ehesten an seiner Beliebtheit abzumessen. In der Antike schon waren auch die schmerzstillenden Eigenschaften des Schlafmohns und des Tollkrautes bekannt. Das Opium wurde aber wegen seiner Giftigkeit bis in die zweite Hälfte des 17 . Jahrhunderts nur sehr zurückhaltend angewen$\operatorname{det}^{20}$. Aus Gründen, die vermutlich mit der Verweltlichung der Religionslehren zusammenhängen, bekam die pharmakologische Schmerzbekämpfung erst im 18. und 19. Jahrhundert größere Bedeutung. Im 19. Jahrhundert wurden dann dank der Fortschritte der Chemie die ersten Schmerzmittel chemisch dargestellt. Die Reihe wird 1880 mit Phenacetin eröffnet und wurde seit den 60 er Jahren des 20 . Jahrhunderts durch die sog. Tranquillizers bereichert. Theorien über deren Funktionsweise, wie die erwähnte Enkephalintheorie, datieren in die neueste Zeit. Es bleibt darauf hinzuweisen, daß die Bekämpfung schwerer Schmerzzustände und vor allen auch des elusiven Schmerzes heute noch große Schwierigkeiten bereitet. 


\section{Bemerkungen zum Stellenwert des Schmerzes in der Mentalität}

\section{Religion}

Wir haben eingangs vermutet, daß die eigenartige Absenz der Schmerzbetrachtung in ärztlichen Kreisen vor 1550 u. a. auch darauf beruhe, daß der Schmerz kein eigentlich medizinisches Problem war, sondern vielmehr in die Zuständigkeit der Kirche gefallen und Gegenstand religiöser Übung gewesen sei.

Ganz allgemein kann gesagt werden, daß der Schmerz traditionell in die christliche Theologie als die gerechte Strafe Gottes für den sündigen Menschen integriert war. Die biblische Figur des Hiob diente als Veranschaulichung des von Gott unbegreiflich geschlagenen Menschen. In Anschauung solcher Leiden und derjenigen verschiedener Märtyrer lehrte die Religion das Erdulden und Ertragen, das Hinnehmen auch schwerster und andauernder Schmerzen. Es darf gesagt werden, daß die Kirchen heute in ihrer seelsorgerischen Tätigkeit ihre Lehre allenfalls modifiziert, aber nicht grundsätzlich verändert haben.

Eine spezifische Abwandlung dieser Mentalität zeigt sich in der seit dem 17. Jahrhundert aufkommenden pietistischen Bewegung. Während für die römisch-katholische und die orthodoxen Kirchen die Auferstehung Christi und somit Ostern das zentrale Thema der Christologie bedeutet, wurde es für die protestantischen Kirchen unter pietistischem Einfluß immer mehr der Karfreitag, der Höhepunkt der Schmerzen Christi. (Auch die kirchlichen Feiern zu diesem Gedenktag mußten eine neue, den anderen Bekenntnissen unbekannte liturgische Gestaltung erhalten. Es wäre der Untersuchung wert, die Karfreitagsliturgie auf ihren Gehalt an Schmerzvokabular vor und nach der Reformation zu untersuchen, wobei zu vermuten ist, daß hier das speziell protestantische Verständnis von Schmerz ebenfalls nachzuweisen wäre.)

Der Pietismus, der bekanntlich den größten Wert auf die individuelle Frömmigkeit legte, forderte von seinen Anhängern die tägliche Betrachtung der eigenen Sündhaftigkeit und Hinfälligkeit. Damit wurde ein Anreiz geschaffen, durch Introspektion auch der eigenen Schmerzen inne zu werden, die dann wiederum in Beziehung zum Passionsgedanken relativiert werden konnten. Wie aus einigen der zahlreichen Briefwechsel aus pietistischem Milieu hervorgeht, wurde der Schmerz auf diese Art zum Mittel der Religionsübung und kultivierte zu diesem Zwecke geradezu eine gewisse hypochondrische Wehleidigkeit. 
Zuvor wurde erwähnt, daß die pharmakologische Schmerzbekämpfung ihre Zurückhaltung erst aufgab, als sich die Verweltlichung der Religionslehren abzuzeichnen begann. Setzt man nun dieses in Parallele zur gleichzeitig vorhandenen Tendenz der Schmerzbetrachtung durch religiöse Introspektion, so muten solche pietistischen Bestrebungen an wie ein Versuch, die schon im Begriffe stehende Schmerzbewältigung durch weltliche Mittel eben Opium - im Schach zu halten.

Besonders hartnäckig bekämpften die Kirchen die Linderung des Geburtsschmerzes. Die Legitimation bezogen sie aus dem Alten Testament («Mit Schmerzen sollst du deine Kinder gebären»). Diese Haltung dürfte aber einen nicht geringen Teil Frauenfeindlichkeit verbergen. Säkularisiert begegnen wir dieser Einstellung noch in der Ansicht, daß nur ein kräftiger Geburtsschmerz die wahre Mutterliebe verbürge ${ }^{21}$.

Wie im Gebiet der Krankheitslehre und in der Nervenforschung zuvor zeigt sich auch in der Religion eine Verdinglichung bzw. Verweltlichung des Schmerzgeschehens an. Heute hat der Schmerz eine Stelle zwischen Transzendenz und Immanenz inne.

\section{Gesellschaft}

Die mit Locke eingeleitete Bewußtwerdung der Sensation entwickelte sich im 18. Jahrhundert zur Sensibilität und wuchs schließlich zum «sentiment», zur Empfindlichkeit. Man kann dazu aus fast allen Lebensgebieten Beispiele beibringen. In der Religion wendet sich, wie angedeutet, der Pietismus und die entsprechenden Tendenzen in Frankreich gegen die institutionalisierten Kirchen und stützen die empfindsame individuelle Frömmigkeit. In der Dichtung bahnt sich der romantische Subjektivismus an. Durch die Malerei wird die Sensibilität für das Landschaftserleben erhöht. Das Individuum, das je verschieden empfindet, gewinnt ganz allgemein an Bedeutung, eine Entwicklung, die in der Deklaration der Menschenrechte während der französischen Revolution gipfelt. Im gesellschaftlichen Verkehr werden die Empfindungen offen geäußert. Man weint, man umarmt sich, erzählt seine Glücksgefühle, ist aber auch für den Lebensüberdruß empfindlich. Wie empfände man in einer solchen Zeit nicht auch die Krankheit und den Schmerz, vor allem den elusiven Schmerz? In diesem Zusammenhang ist es auch zu sehen, daß die Krankheit Hysterie/ Hypochondrie im 18. Jahrhundert einen so hervorragenden Platz einnimmt ${ }^{22}$. 
Die Aufmerksamkeit, welche diese «interessante» Krankheit gewinnt, ist wahrscheinlich darauf zurückzuführen, daß für breitere, vor allem aber die oberen Schichten in einem, vielleicht seit der Augusteischen Zeit nicht mehr dagewesenen Ausmaß Muße entstand. Von England ausgehend, breitete sich zunächst das Manufakturwesen, dann der frühe Industrialismus über Europa aus und verschaffte die nötige Zeit, daß sich ein nicht unbeträchtliches Publikum vom Schmerz plagen lassen konnte.

Auf eine andere Art wurde ebenfalls Zeit gewonnen. Es darf ohne Übertreibung gesagt werden, daß seit 1347 - dem erstmaligen Auftreten der Bubonenpest - bis etwas nach 1700 das Krankheitsgeschehen in derart ausgeprägtem Maß von den vielen hundert Pestepidemien beherrscht war, daß das Krankheits- und Schmerzerleben ganz vorwiegend ein Erlebnis des plötzlichen Todes gewesen war. Aus Gründen, die hier nicht näher zu untersuchen sind, begannen die Pestepidemien nach 1650 abzuklingen. Seuchen traten hernach gewiß noch auf, aber nicht mit dieser verheerenden Tödlichkeit. Seit den ersten Jahrzehnten des 18. Jahrhunderts zeichnete sich außerdem der Erfolg der ersten tatsächlich wirksamen Vorbeugung gegen die Pocken in Form der Impfung ab. Zwischen Krankheitsbeginn und Krankheitsende tritt demnach ein Intervall; die Zeit dehnt sich, man gewinnt «Muße», sich mit dem Krankheitsgeschehen auseinanderzusetzen. Indem der Tod einen kleinen Schritt zurückweicht, findet sich Platz für den Schmerz.

Der Schmerz, allerdings etwas Unangenehmes, fand dergestalt durch das 18. Jahrhundert seinen Wert, ja seinen Unterhaltungswert. Das vom Schmerz geplagte Publikum förderte eine Marktlücke zutage, die durch eine immer besser organisierte medizinische Scharlatanerie ausgefüllt wurde. Zu erwähnen ist als rasch aufkommende und vorübergehende chinesische Mode die Akupunktur, der Brownianismus und schließlich der Magnetimus des Anton Mesmer, der wohl am deutlichsten zeigt, wie hysterisch/hypochondrische Patienten mit ihrerseits hysterisch/theatralischen Darstellungsmitteln kuriert oder mindestens temporär befriedigt werden konnten.

Im 19. Jahrhundert, der viel pragmatischeren Periode der Industrialisierung, fand der Bürger keine Zeit mehr für den Schmerz. Im Maße, wie die Frau des Bürgers zur schönen Müßiggängerin stilisiert wurde, fand sie Zeit für den elusiven Schmerz. So war es denn vor allem die Frau, welche Sigmund Freud seit 1890 die Beobachtungsgrundlage lieferte, anhand deren er seine psychoanalytischen Konzepte entwickeln konnte. 


\section{Überblick}

Zieht man heute eine vorläufige Bilanz über die Rolle des Schmerzes in der Pathologie, Neurophysiologie und Mentalitätsgeschichte, könnte sie folgendermaßen aussehen. Der Schmerz wurde aus dem abgerundeten Weltverstehen, wie es sich bis 1550 darstellte, entlassen. Ganz allgemein stellt man eine Verdinglichung der Schmerzauffassung fest. Einerseits fand das Phänomen Schmerz in der Krankheitslehre seinen Ort im pathologisch veränderten körperlichen Substrat, andererseits wurde es von der Pathophysiologie des Nervensystems durch die Gate-Control-Theorie eingegrenzt. Die Schmerzbekämpfung kann das Geschehen, allerdings auf Kosten der psychischen Klarheit und Präsenz pharmakologisch weitgehend beherrschen. Parallel zur Verdinglichung verläuft in der Mentalitätsgeschichte die Verweltlichung des Schmerzes, wobei auch eine gewisse Kommerzialisierung noch näher zu untersuchen wäre.

Es bleibt indessen ein Rest übrig, weil die Gesellschaft vor dem von uns elusiv genannten Schmerzgeschehen verhältnismäßig ratlos ist. Die Schulmedizin konstatiert in jüngerer Zeit ein wachsendes Interesse an unorthodoxen Heilmethoden und versucht, diesem mit der psycho-somatischen Ausrichtung der Medizin entgegenzutreten. Man stellt dabei fest, da $\beta$ es nicht so sehr der Schmerz ist, der solche Patienten plagt, sondern die Angst vor Vernichtung und Tod. Damit kommen wir zum Schnittpunkt, wo sich Immanenz und Transzendenz des Schmerzgeschehens und Erlebens treffen, und wir befinden uns vor der neuen Situation, in welcher der verdinglichte und verweltlichte Schmerz in ein wieder umfassendes Weltverstehen integriert werden sollte.

\section{Anmerkungen}

${ }^{1}$ Peter Roth, De Dolore, Diss. med. Vorsitz Theodor Zwinger I Basel 1583, Thesis IV

2 ibid. Thesis XXIII

${ }^{3}$ Felix Platter, Praxeos seu de cognoscendis, praedicendis, praecavendis curandisque affectionibus homini incommodantibus tractatus, De Doloribus, Basel 1603

${ }^{4}$ Thomas Sydenham, Dissertatio epistolaris ad ... Gulielmum Cole, M. D. de ... affectione hysterica, London 1685, 66/67, in: Opera omnia, London 1844.

${ }^{5}$ ibid. 60

${ }^{6}$ ibid. 72

7 ibid. $79 / 80$

${ }^{8}$ François Boissier des Sauvages, Nosologia methodica, Amsterdam 1763 
${ }^{9}$ William Cullen, Apparatus ad nosologiam methodicam ..., 5. Auflage, Amsterdam 1775

${ }^{10}$ Dictionnaire des sciences médicales, Stichwort: Nevralgie, Paris 1812-1822

${ }^{11}$ Rudolf August Vogel, Definitiones generum morborum Göttingen, 1764

12 Johann Baptist Michael Sagar, Systema morborum systematicum ..., Wien 1771

${ }^{13}$ K. D. Keele, Anatomies of Pain, Oxford 1957

${ }^{14}$ loc. cit. No 1, Thesis VII et VIII

${ }^{15}$ René Descartes, Discours de la méthode, viele Ausgaben

${ }^{16}$ John Locke, An Essay Concerning Human Understanding II/1 ed: Everyman, London/New York 1971

${ }^{17}$ Ursula Bueck-Rich, Ernst Heinrich Weber (1795-1878) und der Anfang der Physiologie der Hautsinne; Diss. med. Zürich 1970

${ }_{18}$ Thomas Willis, Cerebri anatome, London 1664

${ }^{19}$ loc. cit. No 13

${ }^{20}$ Ulrich Tröhler, Pain: Historical Changes in the Therapeutic Attitude towards, and Physiological Explanation of a Pathological Symptom, in: T. Ogawa (Hrsg.) Pathology, Tokyo, The Taniguchi Fondation, Division of Medical History (im Druck)

${ }^{21}$ Mündliche Mitteilung von Prof. C. Haffter

${ }^{22}$ Esther Fischer-Homberger, Hypochondrie, Bern 1970

\section{Literatur}

K. Akert, Vergangenheit und Zukunft des menschlichen Gehirns, in: NZZ Nr. 100, 30.4. 1984

A. C. Crombie: Early Concepts of the Senses and the Mind, in: Scientific American, 210/5/1964

F. Garrison, History of Neurology, Springfield/Ill. 1957

C. Habrich, Pathographische und aetiologische Versuche medizinischer Laien, in: Heilberufe ..., ed. Echardt und Geier-Kordesch, Münster 1982

C. Haffter, Begriff der Zivilisationskrankheit, in: Gesnerus 36/1979/3.4

H.-R. Isler, Thomas Willis, Stuttgart 1965

R. Janzen (ed.), Schmerzanalyse, Stuttgart 1981

H.M.Koelbing, Operationsschmerz, Anästhesie, Anästhesiologie, in: 30 Jahre Gesellsch. f. Anästhesiologie 1982

L.S. King, Medical World of the 18th Century, Huntingdon/N. Y. 1958

W. Larbig, Schmerz, Stuttgart 1982

K.E. Rothschuh, Geschichte der Physiologie, Berlin/Göttingen/Heidelberg 1953

K.E. Rothschuh, Geschichtliches zur Physiologie des Schmerzes, in: Documenta Geigy, Basel 1965

H. Schipperges, Kleine Kulturgeschichte der Haut, in: Ruperto Carola 43.44/1968/206

H. Schipperges, Pathos und Schmerz, in: Was ist theoretische Pathologie, Berlin/Heidelberg/New York 1979

C. B.Schmid, Der Schmerz in der klinischen Medizin des frühen 19. Jahrhunderts ..., Diss. med. Zürich 1976

M. Schmidt, Pietismus, Stuttgart 1972

C. Sherrington, The Endeavour of Jean Fernel, London 1948

J.Steudel, Bau und Funktion der Haut in der Antike, in: Studium Generale, 17, 1964 


\section{Zusammenfassung}

Um 1550 wird das Phänomen Schmerz Thema eigenständiger Untersuchungen, was sich alsbald in der Krankheitslehre und in der Erforschung des Nervensystems niederschlägt. In der Geschichte der Krankheitslehre bis 1800 wird gezeigt, daß der Schmerz aus der dominierenden Stelle, die er um 1600 gewonnen hatte, in seine heutige Bedeutung als Krankheitssymptom abgleitet. Im Abschnitt über die Physiologie des Nervensystems wird die Entwicklung der Verortung des Schmerzgeschehens nachskizziert. Schließlich folgt eine kurze Darstellung der Verweltlichung des Schmerzes trotz der Religion und seine größere Beachtung dank erhöhter Muße im 18. Jahrhundert.

\section{Summary}

Around 1550, pain is developping into a research-topic of its own. Soon thereafter, the importance of pain in pathology and physiology of the nervous system was reassessed. In the history of pathology up to 1800 , we find that pain has receded from its previously dominant position in 1600 to the mere symptom of disease, which is has guarded up to the present day. In the physiology of the nervous system pain gradually assumed localisation in perpheral and central structures. In religion and society we witness a secularisation and growing importances of pain, due also to greater leisure in the 18th century.

Frau Dr. med. Antoinette Stettler

Kirchenfeldstraße 45

CH-3005 Bern 